INPLASY

PROTOCOL

To cite: Heidari et al.

Disparities in Usage of

Telemedicine in Era of

CoVid-19: A Scoping Review

Protocol. Inplasy protocol

2021100053. doi:

10.37766/inplasy2021.10.0053

Received: 16 October 2021

Published: 16 October 2021

Corresponding author:

Afshin Heidari

afshin.heidari@live.com

Author Affiliation:

Isfahan University of Medical

Sciences.

Support: None.

Review Stage at time of this submission: Piloting of the study selection process.

Conflicts of interest:

None declared.

\section{Disparities in Usage of Telemedicine in Era of CoVid-19: A Scoping Review Protocol}

Heidari, $\mathrm{A}^{1}$; Biroudian, S2; Zamanian, $\mathrm{H}^{3}$.

Review question / Objective: This study aims to distinguish disparities in usage and telemedicine access in the era of CoVid-19 from diverse perspectives.

Condition being studied: Telemedicine is the distribution and delivery of health services through remote telecommunications. Since the beginning of the CoVid-19 Pandemic, the application of telemedicine services has been grown; and by increasing the usage of telemedicine, the access gaps of telemedicine are multiplied. The disparities of telemedicine access and use are being reviewed and discussed in this review.

Information sources: Pubmed, Embase (via Ovid), Psychinfo (via Ovid), WHO Global Index Medicus, Global Health (via Ovid), Cochrane CENTRAL Register of Controlled Trials, Web of Science, SCOPUS, and CINAHL(EBSCO).

INPLASY registration number: This protocol was registered with the International Platform of Registered Systematic Review and Meta-Analysis Protocols (INPLASY) on 16 October 2021 and was last updated on 16 October 2021 (registration number INPLASY2021100053).

\section{INTRODUCTION}

Review question / Objective: This study aims to distinguish disparities in usage and telemedicine access in the era of CoVid-19 from diverse perspectives.
Condition being studied: Telemedicine is the distribution and delivery of health services through remote telecommunications. Since the beginning of the CoVid-19 Pandemic, the application of telemedicine services has been grown; and by increasing the usage of 
telemedicine, the access gaps of telemedicine are multiplied. The disparities of telemedicine access and use are being reviewed and discussed in this review.

\section{METHODS}

Search strategy: We had different search strings for various databases, with the same foundation. The fundamental search strategy of our review was: (Telemedicine OR tele* OR online OR digital* ${ }^{\star}$ OR virtual OR "Mobile Applications" OR smartphone* OR mobile OR cellphone OR app OR application* $^{*}$ OR software* ${ }^{*}$ OR web OR

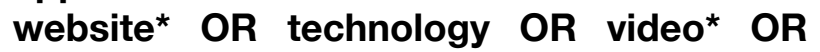
computer* OR ehealth OR e-health OR mhealth OR telephone* OR phone* OR remote OR distance OR "no-show visits" OR "artificial intelligence" OR ai) AND (disparit * OR discrepancy* OR inconsistency OR imbalance OR inequalit* OR unevenness OR disproportion OR variance OR variation OR divergence OR polar* OR equity OR fair* OR justic* OR impartial* OR integrity OR inequity OR unequal* $^{*}$ OR "healthcare disparities" OR "health status disparities" OR availability OR fair* OR "progress-plus" OR divid*) AND (covid-19 OR sars-cov-2 OR 2019ncov OR "corona virus" OR coronavirus OR "Wuhan coronavirus" OR "novel coronavirus" OR ncov-2019).

Participant or population: The studies which evaluated telemedicine use or access in any population (considering different groups disparities) will be included since the beginning of the CoVid-19 pandemic.

Intervention: As a scoping review, there is no intervention in this study, and we'll review every type of telemedicine usage.

Comparator: There is no comparator in this study.

Study designs to be included: All records, regardless of the study design, will be included.

Eligibility criteria: *Focus on any disparities such as gender-based, and socioeconomic status ${ }^{\star} \mathrm{Be}$ related ad done in the CoVid-19 era*Focused on the use of telemedicine, telehealth, mhealth, and e-health in any known form*All articles, regardless of the study design -such as editorial- met other inclusion criteria*Studies that have a statistical comparison between at least one type of disparities between two groups or more.

Information sources: Pubmed, Embase (via Ovid), Psychinfo (via Ovid), WHO Global Index Medicus, Global Health (via Ovid), Cochrane CENTRAL Register of Controlled Trials, Web of Science, SCOPUS, and CINAHL(EBSCO).

Main outcome(s): Disparities in any of these categories: racial and ethnical, income, age, language, gender, payer/ insurance, educational level, marital status, employment status, residence status, housing, specific functional impairments, sexual identity, degree, geographic region, etc.

Data management: We developed various search strings for different databases, and the results were gathered in our record pool and exported to EndNote ver. 20. After removing duplicates, the records were extracted to the Rayyan website. Now on, two independent reviewers will screen articles in two stages (abstract and title screening and full-text screening). Then data extraction will be performed by two independent reviewers into a pre-designed data extraction form in Microsoft Excel. Any conflicts in screening or data extraction will be resolved by consensus or third reviewer opinion. The extraction from columns is Title, First Author, Journal, Year, Study Design, Publication Type, subjects characteristics, the definition of telehealth, statistical parameters, and disparities categories. We developed various search strings for different databases, and the results were gathered in our record pool and exported to EndNote ver. 20. After removing duplicates, the records were extracted to the Rayyan website. Now on, two independent reviewers will screen articles in two stages (abstract and title screening and full-text screening). Any 
conflicts in screening or data extraction will be resolved by consensus or third reviewer opinion. Then data extraction will be performed by two independent reviewers into a pre-designed data extraction form in Microsoft Excel. The extraction from columns is Title, First Author, Journal, Year, Study Design, Publication Type, subjects characteristics, the definition of telehealth, statistical parameters, and disparities categories.

Quality assessment / Risk of bias analysis: Two independent authors will evaluate the quality of cohort studies with the Newcastle Ottawa Scale (NOS) for cohorts, and randomized controlled trial studies with the Newcastle Ottawa Scale (NOS) for RCTs, and cross-sectional studies with the modified Newcastle Ottawa Scale (NOS) for cross-sectional studies.

Strategy of data synthesis: Since it's a scoping review and we will have no metaanalysis, we will perform just qualitative synthesis considering different categories of disparities; such as income, gender identity, insurance type or payer.

Subgroup analysis: None.

Sensitivity analysis: None.

Country(ies) involved: Iran.

Keywords: Telemedicine, Disparity, CoVid-19, Systematic Review.

Contributions of each author:

Author 1 - Afshin Heidari.

Email: afshin.heidari@live.com

Author 2 - Saeed Biroudian.

Email: biroudian.s@iums.ac.ir

Author 3 - Hadi Zamanian.

Email: hadi_zamanian@yahoo.com 